\title{
BMJ Open Study protocol for Chronic Obstructive Pulmonary Disease-Sitting and ExacerbAtions Trial (COPD-SEAT): a randomised controlled feasibility trial of a home-based self-monitoring sedentary behaviour intervention
}

\author{
Mark Orme, ${ }^{1}$ Amie Weedon, ${ }^{2}$ Dale Esliger, ${ }^{1,3}$ Paula Saukko, ${ }^{2}$ Mike Morgan, ${ }^{4}$ \\ Michael Steiner, ${ }^{1,4}$ John Downey, ${ }^{3}$ Sally Singh, ${ }^{1,4}$ Lauren Sherar ${ }^{1,2}$
}

To cite: Orme M, Weedon A, Esliger D, et al. Study protocol for Chronic Obstructive Pulmonary Disease-Sitting and ExacerbAtions Trial (COPDSEAT): a randomised controlled feasibility trial of a home-based self-monitoring sedentary behaviour intervention. BMJ Open 2016;6:e013014.

doi:10.1136/bmjopen-2016013014

- Prepublication history for this paper is available online. To view these files please visit the journal online (http://dx.doi.org/10.1136/ bmjopen-2016-013014).

SS and LS are joint last authors.

Received 13 June 2016 Revised 3 September 2016 Accepted 5 September 2016

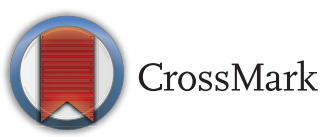

For numbered affiliations see end of article.

Correspondence to

Mark Orme;

m.w.orme@|boro.ac.uk

\section{ABSTRACT}

Introduction: An acute exacerbation of chronic obstructive pulmonary disease (COPD) marks a critical life event, which can lower patient quality of life and ability to perform daily activities. Patients with COPD tend to lead inactive and highly sedentary lifestyles, which may contribute to reductions in functional capacity. Targeting sedentary behaviour (SB) may be more attainable than exercise (at a moderate-tovigorous intensity) for behaviour change in patients following an exacerbation. This study aims to evaluate the feasibility and acceptability of a 2-week at-home intervention providing education and self-monitoring to reduce prolonged periods of $S B$ in patients with COPD discharged following an acute exacerbation.

Methods and analysis: Patients will be randomised into 1 of 3 conditions: usual care (control), education or education+feedback. The education group will receive information and suggestions about reducing long periods of sitting. The education+feedback group will receive real-time feedback on their sitting time, standups and step count at home through an inclinometer linked to a smart device app. The inclinometer will also provide vibration prompts to encourage movement when the wearer has been sedentary for too long. Data will be collected during hospital admission and 2 weeks after discharge. Qualitative interviews will be conducted with patients in the intervention groups to explore patient experiences. Interviews with healthcare staff will also be conducted. All data will be collected January to August 2016. The primary outcomes are feasibility and acceptability, which will be assessed by qualitative interviews, uptake and drop-out rates, reasons for refusing the intervention, compliance, app usage and response to vibration prompts.

Ethics and dissemination: The research ethics committee East Midlands Leicester-Central has provided ethical approval for the conduct of this study. The results of the study will be disseminated through appropriate conference proceedings and peer-reviewed journals.

\section{Strengths and limitations of this study}

The study will target a critical period for behaviour change in a clinical population admitted to hospital.

- Important insights into the suitability of focussing on sedentary behaviour (SB) using novel wearable technology will be obtained.

- Given the timing of the intervention, it will not be possible to obtain an objective assessment of habitual physical activity or SB prior to hospital admission.

Trial registration number: ISRCTN13790881; Pre-results.

\section{INTRODUCTION}

Targeting increases in physical activity in patients with chronic obstructive pulmonary disease (COPD) has been the emphasis of a large number of exercise training and behaviour change interventions for over a decade. ${ }^{1}$ Despite these considerable efforts, there has been limited success. ${ }^{1}{ }^{2}$ The lower levels of physical activity coupled with the often fragile physical and psychological health (eg, low exercise capacity and low self-esteem) among patients with COPD may make reducing sedentary behaviour $(\mathrm{SB})$ a more suitable conduit for behaviour change. ${ }^{3}{ }^{4} \mathrm{SB}$ is defined as 'any waking behaviour characterised by an energy expenditure $\leq 1.5$ metabolic equivalents while in a sitting or reclining posture'. Patients with COPD demonstrate significantly higher levels of SB compared with healthy controls. ${ }^{6}$ 
An acute exacerbation of COPD marks a critical life event, characterised by a worsening of symptoms beyond normal day-to-day variation; bringing with it a plethora of negative impacts affecting both physical and psychological health. ${ }^{7}$ For example, exacerbations contribute to reductions in patients' abilities to perform activities of daily living. ${ }^{8}$ For patients hospitalised for an acute exacerbation of COPD, there is amplitude attention at the initial phase of admission followed by $\sim 7$ days of monitored inactivity and sedentariness. ${ }^{9}$ Consequently, patients may be discharged less well equipped to manage their 'usual routine' than when they were admitted. ${ }^{9}$ Interventions for patients with COPD in close temporal proximity to hospital admissions are promising, with pulmonary rehabilitation within 4 weeks postdischarge, found to reduce rehospitalisation in the preceding 3 months. ${ }^{10}$ Despite this, postdischarge pulmonary rehabilitation is sparsely taken up by patients who are offered it at the point of discharge. ${ }^{11}$ A stepping stone approach which does not emphasise exercise and does not require travel and additional time/appointments may serve to facilitate the physical and psychological well-being required for patients to invest in pulmonary rehabilitation. To date, few studies have specifically targeted non-rehabilitation physical activity or SB immediately after discharge. There is a need to determine the feasibility and acceptance of such programmes, the evaluation of which may be enhanced through qualitative approaches. Such an approach may even offer as an alternative for those patients unable to exercise at sufficient capacity to reduce readmission risk. ${ }^{12}$

The time between hospital discharge and the initiation of pulmonary rehabilitation (for those who attend) marks an important period for patients. Pitta $e t a l^{13}$ highlighted that patients with low activity levels at 1 month after discharge were more likely to be readmitted within the following year. Therefore, there is a need for behavioural interventions for patients on leaving the hospital in order to prevent further decline in quality of life, functional capacity and potentially encourage uptake of pulmonary rehabilitation. Owing to the impact of an acute exacerbation (eg, dyspnoea and fatigue) interventions requiring large amounts of effort or time may be impracticable. ${ }^{11}$ Consequently, targeting SB (eg, long periods of consecutive sitting) during the early stages of postdischarge recovery may act as a catalyst to encourage patients to sit less and move more; equipping them with the ethos and habits to better engage in pulmonary rehabilitation when the time comes. Furthermore, targeting the displacement of SB with light activity such as walking may help to reduce the risk of readmissions. ${ }^{14}$ Indeed, physical inactivity is widely regarded as the strongest predictor of all-cause mortality in $\mathrm{COPD}^{15}$ and preliminary evidence has suggested that a reduction in sedentary time from discharge to 6 weeks will also help reduce the risk of readmissions. ${ }^{16}$

Wearable technologies such as pedometers, which provide a basis for self-monitoring and real-time feedback, have been shown to elicit increases in physical activity in patients with COPD. ${ }^{17} 18$ Patient-driven healthcare, facilitated by behavioural feedback and/or prompting, may empower patients to improve and track their health outcomes. ${ }^{19}$ In a metaregression to identify the active ingredients in activity promotion research, Michie et $a t^{20}$ found self-monitoring and feedback to be among the most potent behaviour change techniques. Coupling behaviour change strategies such as self-monitoring and feedback, which are grounded in control theory, ${ }^{21}$ tend to have an impact greater than the sum of their independent effects. ${ }^{20}$ As supported by a comprehensive review of SB change strategies, ${ }^{22}$ providing patients with education, self-monitoring, real-time feedback and behaviour prompts may yield promising results. However, in postexacerbation care for patients with COPD, the use of wearable technology for behaviour change has not been widely explored. Technology competency and psychosocial issues in this population, particularly during phases of acute illness, makes examining the uptake and engagement with such interventions fundamental to future success. The 'Chronic Obstructive Pulmonary Disease Sitting and ExacerbAtions Trial' (COPD-SEAT) will focus on the feasibility of delivering a home-based SB self-monitoring intervention in patients following hospital discharge for an acute exacerbation.

The objectives of the trial are to:

1. Examine the feasibility of the 'COPD-SEAT' intervention including the trial design, recruitment, adherence and procedures.

2. Assess the acceptability of the intervention among patients receiving the intervention and healthcare staff involve in the care pathway.

3. Reduce prolonged periods of SB at home in patients with COPD admitted for an acute exacerbation.

\section{METHODS AND ANALYSIS}

\section{Trial design and registration}

The feasibility study is a three-armed randomised controlled trial (RCT) with 1:1:1 individual allocation comparing usual care (control) with usual care plus education (education intervention) and usual care with education plus feedback (education+feedback intervention). The design of the study and flow of participants is presented in figure 1.

This study will be conducted, analysed and reported according to the Consolidation Standards of Reporting Trials (CONSORT) statement for cluster RCTs. ${ }^{23}$ The University Hospitals of Leicester will act as study sponsor. The trial has been prospectively registered on the ISRCTN website.

\section{Participants}

Eligibility criteria for patients

Patients eligible for inclusion in the study will be: aged between 40 and 85 years; have a confirmed diagnosis of COPD; have experienced fewer than four exacerbations 


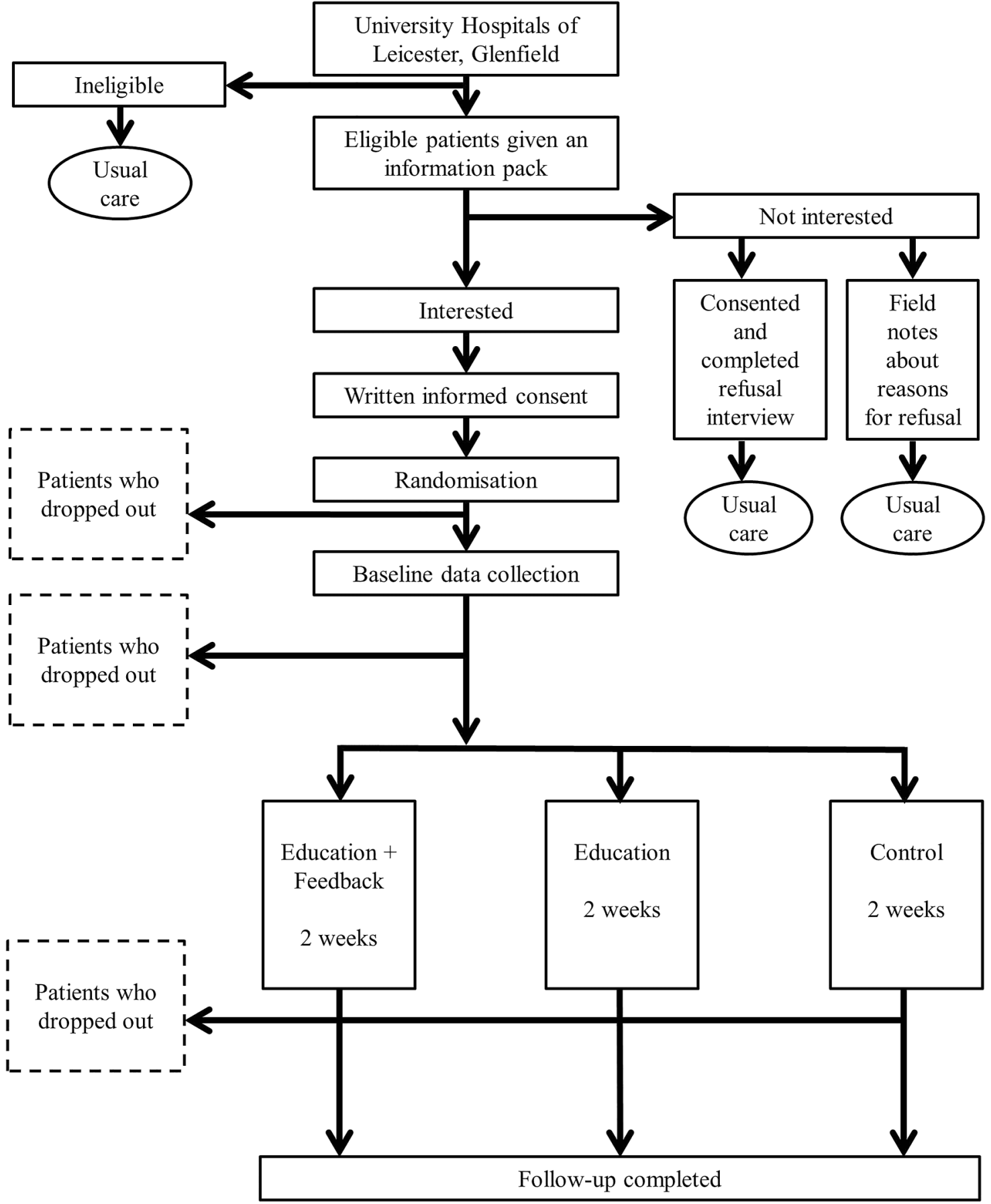

Figure 1 Flow of participants through the trial.

requiring hospital admission in the previous 12 months; have had a confirmed acute exacerbation as the reason for current hospitalisation; willing and able to comply with the trial protocol; are physically able to participate in light intensity physical activity (ie, walking with an aid); and able to provide informed consent (eg, read and understand English).

Patients will be excluded if the COPD specialist nurses or clinicians consider them unsuitable for the project for any reason: for example, patients with severe mental impairment or terminally ill; patients with an injury or additional health condition that precludes their ability to take part in light intensity physical activity; patients with an overlying medical disorder that interferes with provision of consent, completion of measurements, intervention, interview or follow-up; and/or taking part in concomitant research studies.

\section{Setting}

Participants will be recruited from patients admitted to Glenfield Hospital, University Hospitals of Leicester 
NHS Trust for an acute exacerbation of COPD and will be screened for eligibility by COPD specialist nurses.

\section{Procedure}

Eligible patients will be given a verbal description of the study, participant information sheet and expression of interest form by a researcher after they have received usual care from a COPD specialist nurse. A researcher will revisit the patients at the bedside at an agreed time to collect the expression of interest form from the patient. For those patients who are not interested in taking part in the trial, reasons for this (if offered freely) may be taken as field notes or by an interview if patients consent to this. For patients wishing to take part in the study, informed consent will be obtained followed by prerandomised group allocation. If allocated to the education or education+feedback group, patients will then receive the respective intervention. Questionnaires, activity monitor deployment and qualitative interview (intervention groups only) will then be completed prior to discharge. The timing of these procedures will vary based on expected discharge. On discharge, patients will wear the activity monitors for 2 weeks before returning for their follow-up appointment.

\section{Trial interventions}

\section{Control}

Although there are some variations in practice, "usual care' offered to patients with COPD admitted to hospital for an acute exacerbation typically comprises brief advice to take part in regular exercise, information about pulmonary rehabilitation, and a referral to a follow-up clinic if the patient is eligible and does not decline pulmonary rehabilitation during their admission. No in-hospital rehabilitation is conducted as part of usual care. Patients also receive brief discussions and advice regarding smoking cessation (if appropriate), medication and inhaler techniques, and oxygen therapy (if appropriate); generally lasting $15-30 \mathrm{~min}$. All patients will receive a phone call during the first week of discharge, as per usual care. Patients will be provided with a 24-hour number to call if they have any questions during the course of their involvement in the trial.

\section{Intervention}

Education Written information will be provided by an educational booklet, four pages in length, adapted for patients with COPD from 'On Your Feet to Earn Your Seat'. ${ }^{24}$ The 'On Your Feet to Earn Your Seat' booklet was originally developed in accordance with the habit formation model $^{25}{ }^{26}$ and designed using a 'small changes' approach as detailed by Gardner et al. ${ }^{24}$ This booklet ${ }^{24}$ was modified to emphasise simpler behaviours, in this case standing up from a chair, which may be more appealing and attainable to patients with COPD postexacerbation compared with more daunting behaviours such as stair climbing. ${ }^{25}$
The researcher will go through the revised booklet, entitled 'Sit Less, Move More, Live Healthier', with the patient at the bed-side outlining the importance of breaking up long periods of sitting and discussing individualised strategies and opportunities for the patient to do this at home. This booklet contains seven suggestions: leave the house daily; make ad breaks active; standups (eg, when waiting for a bus); tiptoe through the queue; increase your steps, sit to stand with no hands and treat the seat as a treat. For each of these tips, 'handy-hints' are provided to facilitate the adoption of these small changes (eg, offering ways to incorporate them into everyday life). The components of the education delivery are presented in box 1 . In addition to the extensive focus groups and interviews for the original booklet, ${ }^{24}$ the 'Sit Less, Move More, Live Healthier' booklet has been through our Pulmonary and Cardiac Rehabilitation Patient and Public Involvement Advisory group who provided feedback on content, readability and design.

Education+feedback Patients randomised into the education+feedback group will receive the above education intervention (ie, 'Sit Less, Move More, Live Healthier' booklet) plus a wearable device to self-monitor their SB and physical activity as well as provide a behavioural nudge in the form of a vibration prompt. The wearable provides real-time, visual and numeric feedback on time spent sitting, standing and lying down, number of sit-to-stand transitions and step count. It also allows the user to look back at previous days, providing day-to-day comparisons in the form of a bar chart.

Patients will be shown how to wear the device at the bedside and will fit the monitor themselves to ensure the device is correctly adjusted to ensure a secure and snug fit. The device, LUMO (Lumo Bodytech, Palo Alto, California, USA), is a small $(4.15 \times 10 \times 0.8 \mathrm{~cm} ; 25 \mathrm{~g})$ and flexible sensor (figure 2) which is worn around the lower back on a belt. The sensor connects wirelessly via low energy Bluetooth to a mobile application on a smart device provided to patients. The embedded inertial sensors of the LUMO continuously track the amount of time spent lying down, slouching, sitting, standing and transitioning from sitting to standing providing the patients with up-to-date information of their behaviour whenever they want it.

Patients will be trained while in hospital on how to navigate the app (touch screen). Real-time feedback is provided on a mobile app with panels displaying time spent sitting (including proportions of time spend standing and stepping; figure 3A), sit-to-stand transitions (figure 3B) and step count (figure 3C) using low cognitive load graphics and data presented on a daily, weekly and monthly format.

Additionally, the LUMO will alert the patient to break up their sitting time when they have been sitting for 'too long' via gentle vibration. After a full explanation of the LUMO and app, patients will be asked how much consecutive sitting they would want to accumulate before 


\section{Box 1 Intervention components}

\section{Education booklet: components to cover during intervention delivery}

It will be explained that the booklet will provide information to help them to sit less and move more.

It will be explained that sitting for too long can be harmful to their health.

It will be explained that breaking up long periods of sitting can help reduce joint stiffness and pain.

The principle of 'use it or lose it' will be explained in relation to deconditioning.

If deemed appropriate, other tailored examples of benefits to be had will be explained.

The patient will be directed to the 7 suggestions for an active recovery.

If deemed appropriate the first suggestion (leave the house daily) will be discussed.

If deemed appropriate the second suggestion (make TV advert breaks active) will be discussed.

If deemed appropriate the third suggestion (stand up when waiting for something) will be discussed.

If deemed appropriate the fourth suggestion (tiptoe when waiting in a queue) will be discussed.

If deemed appropriate the fifth suggestion (increase your steps) will be discussed.

If deemed appropriate the sixth suggestion (sit to stand with no hands) will be discussed.

If deemed appropriate the seventh suggestion (treat the seat as a treat) will be discussed.

If deemed appropriate additional tailored top tip examples will be discussed.

- Smart device: components to cover during intervention delivery

It will be explained that the LUMO and smart device communicate with each other automatically.

Patients will learn how to lock and unlock the smart device.

- LUMO app: components to cover during intervention delivery

It will be explained that the app provides the patient with information on sitting, standing and stepping.

Patients will be shown where to find their time spent sitting 'today' on the app home screen.

Patients will be shown where to find how many time they have stood up 'today' on the app home screen.

Patients will be shown where to find their step count 'today' on the app home screen.

- Sit time panel: components to cover during intervention delivery

Patients will be shown how to access the pie chart for time sitting, standing, stepping and lying down.

Patients will be shown the hourly bar chart for all behaviours.

Patients will be shown how to look back at previous days.

Patients will be shown how to return to 'today's' information.

Patients will be shown how to return to the home screen.

- Stand ups panel: components to cover during intervention delivery

Patients will be shown the hourly bar chart for all behaviours.

Patients will be shown how to look back at previous days.

Patients will be shown how to return to 'today's' information.

Patients will be shown how to return to the home screen.

- Steps panel: components to cover during intervention delivery

Patients will be shown the hourly bar chart for all behaviours.

Patients will be shown how to look back at previous days.

Patients will be shown how to return to 'today's' information.

Patients will be shown how to return to the home screen.

- Vibration prompt: components to cover during intervention delivery

It will be explained that the LUMO device provides a vibration prompt when they have sat for 'too long'.

It will be explained that the vibration will only go off once then the timer will reset.

Patients will choose the duration of consecutive sitting before the vibration occurs.

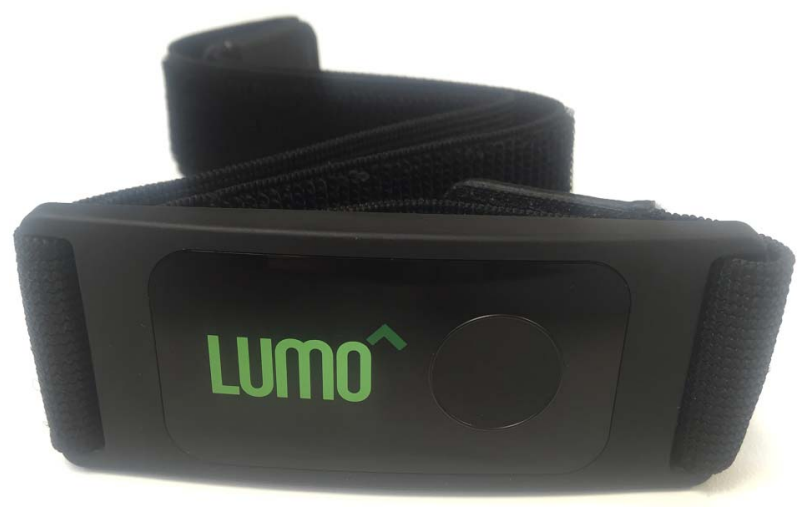

Figure 2 The LUMO sensor. being prompted (eg, every hour of continuous sitting). The vibration frequency will be fixed for the 2-week duration. The components of the LUMO app and vibration prompt delivery are shown in box 1 .

\section{Feasibility}

Measures for assessing feasibility are provided in table 1 and will include: the number of patients eligible for the study; the proportion of patients approached who agree to participate; the duration of the intervention deliveries; views of patients and healthcare staff on the timing of the intervention; and the number of telephone 
contacts and duration of phone calls with patients during follow-up beyond usual care.

\section{Acceptability}

To the patients (education and education+feedback groups only):

A. Patients' experience of the educational intervention in the context of their daily lives and health explored in qualitative interviews (eg, how they found the booklet, eg, did they read or recall it, what aspects they perceived as useful, and how/why and in what contexts they reduced/did not reduce SB, how the intervention could be improved).

B. Proportion of patients who stopped wearing the device during the 2 weeks of follow-up; when they did this (eg, number of days into the follow-up) and why they stopped (assessed during interviews).

C. Proportion of patients who attended the follow-up appointment and reasons why patients did not complete the study (assessed by telephone interview).

To the patients (education+feedback group only):

A. Patients' experience of the LUMO device, mobile application and vibration prompts in the context of their daily lives and health will be explored in the interviews (eg, how they found using the device, their perspectives on the feedback and prompts; how they reduced SB using the device, or why or in what contexts they did not reduce $\mathrm{SB}$, how the technology and feedback could be improved).

B. Patients' response to the vibration prompts, as examined by LUMO data (eg, number of vibrations; average response time from vibration to stand-up; number of times the vibration prompt resulted in behaviour change).

C. Patients' use of the LUMO mobile application, as examined using Flurry app analytics (eg, time spent on the app; time spent in each feedback panel of the app; number of sessions; duration of sessions).

\section{Intervention fidelity}

Examining intervention fidelity may help to explain variance in outcomes (eg, wearing the activity monitors) related to non-adherence or partial adherence to the intervention. ${ }^{27}$ For the education and education+feedback groups, researcher delivery of the intervention (education booklet, LUMO app and vibration prompt) will be audio recorded. The deliveries will be assessed against a checklist of content designed specifically for COPD-SEAT. For example, for the education group, information about why it is important to reduce long periods of sitting should be covered and examples of how to do this discussed. For the education+feedback group, a walk-through of the LUMO app should be conducted with explanations of all information provided by the app (eg, sitting time, stand-ups and step count). This will provide a measure of how much of the intervention was actually delivered to the patients. ${ }^{28}$ Each component of the checklist will be subject to dichotomous scales (present or absent) to determine consistency of delivery and ordinal scales to determine quality of delivery (none, adequate or excellent).

\section{Data collection}

Data will be collected January to August 2016 by trained researchers, following standard operating procedures. Researchers will not be blinded to treatment allocation for qualitative interviews and study measurements. Baseline data will be collected during patients' hospital stay. The timing of baseline data collection cannot be standardised due to variation in the timing of patients receiving usual care from COPD specialist nurses, screening and length of stay.
A
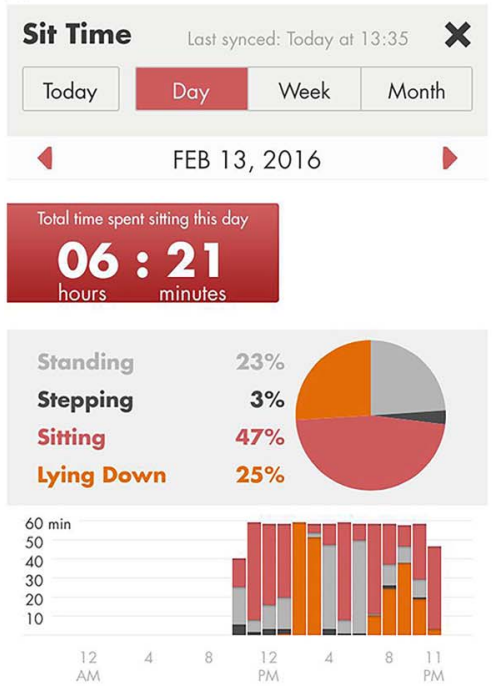

B

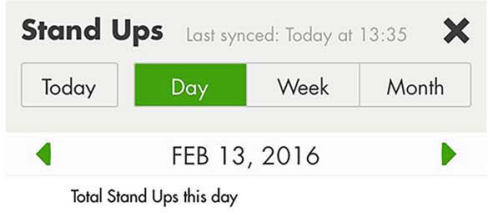

$75_{\text {inimes }}$

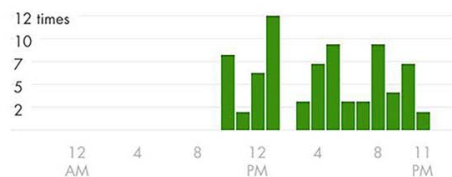

C
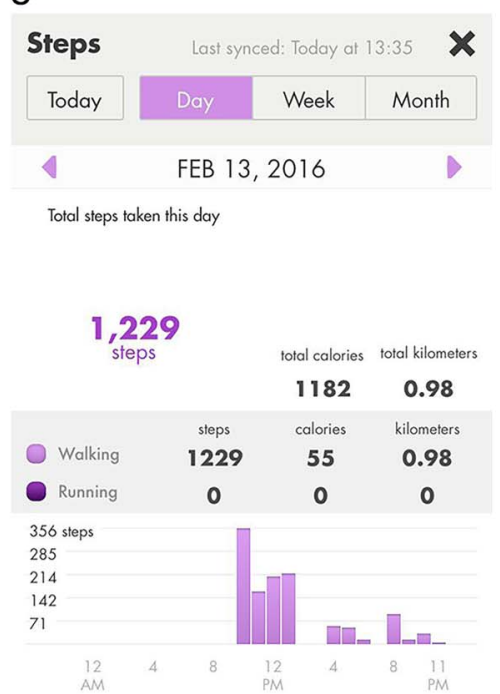

Figure 3 LUMO app panels showing: (A) proportion of the day spent standing, stepping, sitting and lying down; (B) daily number of sit-to-stand transitions; and (C) daily step count. 
Table 1 Outline of feasibility indicators

\begin{tabular}{|c|c|c|}
\hline Indicators & Data sources & Timing \\
\hline \multicolumn{3}{|l|}{ Recruitment } \\
\hline Feasibility of patient screening and recruitment & $\begin{array}{l}\text { Healthcare staff interviews, project records } \\
\text { (eg, number of eligible patients missed) }\end{array}$ & $\begin{array}{l}\text { Ongoing throughout the } \\
\text { study }\end{array}$ \\
\hline $\begin{array}{l}\text { Number of eligible patients, number of patients } \\
\text { screened, number of patients invited to take part, } \\
\text { actual number of patients who consent to take } \\
\text { part }\end{array}$ & Medical records and project records & $\begin{array}{l}\text { Ongoing throughout the } \\
\text { study }\end{array}$ \\
\hline $\begin{array}{l}\text { Number of patients who refuse, drop out or opt } \\
\text { out } \\
\text { Intervention delivery }\end{array}$ & $\begin{array}{l}\text { Patient requests, qualitative patient interviews, } \\
\text { field notes }\end{array}$ & \\
\hline $\begin{array}{l}\text { Duration of intervention sessions (education and/ } \\
\text { or device training); patient engagement in the } \\
\text { content }\end{array}$ & $\begin{array}{l}\text { Audio recordings of the intervention sessions, } \\
\text { qualitative patient interviews }\end{array}$ & $\begin{array}{l}\text { Ongoing throughout the } \\
\text { study }\end{array}$ \\
\hline \multicolumn{3}{|l|}{ Description of unintended events } \\
\hline $\begin{array}{l}\text { It is important to note whether there were any } \\
\text { unintended side effects or outcomes from the } \\
\text { intervention. For example, did patients start } \\
\text { doing one particular activity but at the expense of } \\
\text { another? Unexpected outcomes are not } \\
\text { necessarily negative. For example, there may be } \\
\text { unexpected positive health outcomes. }\end{array}$ & Qualitative patient interviews, medical notes & $\begin{array}{l}\text { 2-week follow-up } \\
\text { appointment }\end{array}$ \\
\hline $\begin{array}{l}\text { Hospital readmissions related to the study. For } \\
\text { example, trying to move more resulting in a fall } \\
\text { Patient satisfaction, acceptability and enjoyment }\end{array}$ & Medical notes & $\begin{array}{l}\text { Ongoing throughout the } \\
\text { study }\end{array}$ \\
\hline $\begin{array}{l}\text { Satisfaction/dissatisfaction with the intervention; } \\
\text { likes/dislikes; suggested improvements }\end{array}$ & $\begin{array}{l}\text { Qualitative patient interviews conducted by } \\
\text { researcher with no involvement in intervention } \\
\text { delivery to encourage honest opinions and } \\
\text { feedback }\end{array}$ & $\begin{array}{l}\text { 2-week follow-up } \\
\text { appointment, analysis of } \\
\text { app usage }\end{array}$ \\
\hline \multicolumn{3}{|l|}{ Potential sustainability } \\
\hline $\begin{array}{l}\text { Whether healthcare staff could envision the } \\
\text { implementation of the intervention within routine } \\
\text { care and what they would alter }\end{array}$ & Qualitative interviews with healthcare staff & $\begin{array}{l}\text { Ongoing throughout the } \\
\text { study }\end{array}$ \\
\hline $\begin{array}{l}\text { The number of telephone contacts and duration } \\
\text { of telephone conversations with patients during } \\
\text { follow-up beyond usual care }\end{array}$ & Project records & $\begin{array}{l}\text { Ongoing throughout the } \\
\text { study }\end{array}$ \\
\hline
\end{tabular}

\section{Sample characteristics}

\section{Objectively measured physical activity and $S B$}

Following hospital discharge (day 0), patients will be asked to wear two monitors, one posture sensor (gyroscope) and one triaxial accelerometer, for 14 days and return the devices at the follow-up appointment (day 15). An ActiGraph wGT3X-BT triaxial accelerometer will be worn on a waistband (on the right anterior axillary line) for 2 weeks to measure time spent in physical activity (ie, sedentary, light, moderate, and vigorous) using common cut-points (eg, as published by Freedson et $a l^{29}$ ) and step count. Patients will be asked to wear the device during waking hours and to only remove it during that period for water-based activities.

The LUMO posture sensor will serve to objectively quantify SB in all groups (control, education and education+feedback). Therefore, it will act as both the measurement and intervention device for the education + feedback group. In addition to physical activity intensity data collected by the ActiGraph accelerometer, the LUMO will provide information on sitting, driving, lying, standing, sit-to-stand transitions and stepping. The device will be worn on the lower back (in contact with skin) for 2 weeks postdischarge. Patients will be asked to wear the monitor during waking hours and to remove it for water-based activities. The LUMO has been found to produce valid measurements of free-living SB (mean error of $9.5 \%)^{30}$ and step count (mean absolute percentage error $0.4 \%)^{31}$ compared with the ActivPAL (PAL Technologies, Glasgow, UK). While no validation study to date has specifically examined LUMO stand-ups, valid free-living SB against the ActivPAL supports accurate detection of sit-to-stand transitions. LUMO data will be analysed in $5 \mathrm{~min}$ epochs with behaviours summarised as a percentage of each 5 min epoch (eg, $50 \%$ sitting will be converted to 2.5 min sitting).

Patients will be asked to wear for the ActiGraph and LUMO monitors concomitantly for two main reasons. First, as noted above, the LUMO monitor is a relatively new device and has not yet been subject to multiple validation studies across different populations (eg, ages, disease states and habitual activity levels). Therefore, the 
ActiGraph provides a well-established measurement of physical activity and sedentary time in COPD and general populations. Second, it is unclear whether patients will dislike the nudge/vibration feature of the LUMO and thus remove it. Consequently there is a need for an independent evaluation and intervention sensor.

\section{Psychosocial health}

During hospital stay and at follow-up a range of health/ psychosocial measures will be taken. COPD-specific health status will be examined using the COPD Assessment Test (CAT; eight items).$^{32}$ Overall health status will be assessed using the EuroQol (EQ-5D-5L; five items).$^{33}$ Additional symptom burdens will be assessed by the Functional Assessment of Chronic Illness Therapy-Fatigue (40 items; FACIT-F) ${ }^{34}$ and the Hospital Anxiety and Depression Scale (HADS; 14 items). ${ }^{35}$ Fear of falling will be assessed using the Falls Efficacy Scale-International (FES-I; 16 items). ${ }^{36}$

\section{Self-reported physical activity and sitting}

During hospital stay patients' usual time spent sitting in domain-specific activities (TV, transport, work, computer and other) during weekdays and weekend days will be self-reported using an adapted version of the Marshall Sitting Time Survey (10 items). ${ }^{37}$ Patients will be asked to complete an adapted version of the International Physical Activity Questionnaire (IPAQ) Short Form (three items) ${ }^{38}$ to assess usual physical activity levels.

\section{Usual dyspnoea severity}

In order to examine patients' usual breathlessness severity, that is, when not acutely ill, the modified Medical Research Council (mMRC) dyspnoea scale (single item $)^{39}$ will be completed.

\section{Body composition and physical function}

At follow-up height, weight and waist circumference will be measured to the nearest $0.1 \mathrm{~cm}, 0.1 \mathrm{~kg}$ and $0.1 \mathrm{~cm}$, respectively. Waist circumference will be measured twice, with a third conducted if the difference between the first two is $>3 \mathrm{~cm}$. Patients will also be asked to complete the Short Physical Performance Battery (SPPB) which comprises repeated chair stands; balance tests and a $4 \mathrm{~m}$ walk. ${ }^{40}$ Upper body skeletal muscle assessment will be grip strength using a hand-held dynamometer.

\section{Sample size estimation and recruitment target}

The study will aim to recruit as many patients as are admitted to hospital for an acute COPD exacerbation within the operational period. One of the main objectives of this feasibility study is to provide data on eligibility and recruitment and to enable an accurate estimation of the required sample size for a future trial based on a realistic recruitment plan. The number of hospital admissions for an acute exacerbation of COPD is 80-100 patients per month and in a previous in-hospital early rehabilitation trial conducted in part at
Glenfield Hospital, overall recruitment uptake was $32.3 \% .^{12}$ Therefore, a similar admissions rate and uptake ( 1 in 3) would be expected for COPD-SEAT.

\section{Randomisation}

Block randomisation (1:1:1) will be used to ensure a balance in sample size between the three study groups. Balanced combinations of group allocations within blocks will be conducted by a researcher at Loughborough University who is independent of the research team. This will ensure study team researchers will be blinded to group allocation prior to patients deciding whether to take part in the study. Owing to limited study team members and logistical barriers, study team researchers will be made aware of group allocation before consent. Patients will only be informed of their group allocation after providing informed consent.

\section{Quantitative data analysis}

As this is a feasibility study, it is not appropriate at this stage to aim for sufficient statistical power to detect changes over time or between group changes. Therefore, quantitative data analysis will primarily be descriptive; in the form of estimations of means and SDs and proportions of patients screened, eligible and willing to participate and complete the trial. In order to examine differences in patient characteristics between study groups, between-group comparisons will be assessed using independent t-tests or analysis of variance (ANOVA). Within-group comparisons will be conducted using paired sample t-tests or repeated measures ANOVA if data meet parametric assumptions.

Data will be entered and housed on a secure webbased database system, Research Electronic Data Capture (REDCap), which has discrepancy management capabilities. Data will be transferred from REDCap to the Statistical Package for the Social Sciences (SPSS) for statistical analysis.

All participant information collected as part of the research will be kept strictly confidential. With permission, patients' general practitioner will be informed of their participation in the study. Any information regarding patients which leaves the hospital will have their name and address removed. Participants will not be identified in any subsequent written materials and results will be reported in such a way that confidentiality is preserved.

\section{Qualitative research}

The aim of the qualitative research is to identify potential barriers and facilitators to participation in the trial and in the self-monitoring and education programme. This information will help maximise chances of avoiding recruitment and retention issues and facilitate optimal qualitative process evaluation of the eventual trial.

Patients randomised to the education or education +feedback intervention groups will be invited to take part in two semistructured interviews. The first interview will 
take place at the bedside before being discharged from hospital and will explore areas including perceptions of $\mathrm{SB}$ in general and in relation to managing COPD, previous experiences of using technology, previous experiences of advice about COPD self-management as well as their study expectations. The second interview will take place at the 2-week follow-up appointment. These interviews will explore the overall acceptability and experiences of the educational and self-monitoring interventions, how the interventions are perceived and how they fit into the daily life and health context of the participants, participants' perspectives on the recruitment process, context and time (ie, following exacerbation) of the intervention (both education and education + feedback) and specific components of the intervention (eg, prompts). Patients, who do not attend their follow-up appointment, will be invited for a telephone interview to explore reasons for not completing the study.

It is not only important to understand reasons for participant engagement with an intervention but also reasons for non-engagement. For patients who are offered the intervention but who do not take part in the main trial, reasons for not wanting to engage with the study will be noted as part of field notes or qualitative refusal interviews where patients consent to this.

Given the timing and setting of the trial (in-hospital and immediately after discharge) qualitative interviews with healthcare staff within the care pathway of hospitalised patients with COPD (eg, clinicians, COPD specialist nurses and ward nurses) will be conducted to explore topics, such as perspectives on the feasibility of reducing SB in this population and in the hospital setting and the use of self-monitoring technology to achieve this. All interviews will be conducted by a social scientist with experience in conducting qualitative research.

\section{Qualitative data analysis}

The qualitative interviews will be recorded, transcribed and analysed using thematic analysis. The approach identifies emerging, recurrent themes or patterns in the interviews. ${ }^{41}$ In the context of this feasibility study, qualitative analysis will mean identifying emergent themes to highlight features of the study, intervention and participants' context that facilitate or hinder their engagement with the trial and the intervention. ${ }^{42}$

All qualitative interviews will be audio recorded (with informed consent) and transcribed in verbatim. Transcripts will be analysed using constant comparison, which identifies recurrent themes in the interviews, initially using free coding and subsequently interrogating emergent themes, particularly those pertaining (but not limited) to the issues facilitating or hindering engagement with the study and intervention. ${ }^{43}$ Following the principles of thematic analysis, emergent themes will be identified and probed throughout the study, so that the interview schedule and coding schedule are modified to follow new leads until new themes no longer emerge. Recruitment of participants will also continue until new themes no longer emerge and the material begins to cohere around key themes, that is, thematic saturation is reached. ${ }^{43}$ The principle of the analysis is to create a coding frame that 'fits' the data rather than trying to make the data 'fit' a pre-existing coding frame to allow examining issues around the intervention from a broad contextual perspective. ${ }^{43}$

\section{ETHICS AND DISSEMINATION}

Findings from this feasibility study, particularly patient preferences and acceptability, will inform the design of any subsequent RCT. The primary outcome for future efficacy trials will be readmission rates. Beyond this, we will seek to implement the findings of the RCT into routine care and locally we will share these results with local secondary care interface groups such as service managers. Results will be disseminated to our Pulmonary and Cardiac Rehabilitation Patient and Public Involvement Advisory group whose members are current and former pulmonary and cardiac rehabilitation patients or interested members of the public who have participated in previous research trials. We have a number of national and international visitors who come to share our practice which we anticipate will stimulate interest in the study findings. We will be able to use the internet to widely publicise the findings of our work and promote interest in the self-monitoring programme.

The results of this feasibility study will be presented to participants of the feasibility study through a dedicated dissemination evening; at appropriate national, international and regional respiratory, physiotherapy and physical activity conferences; local study days in secondary care; and through peer-reviewed journals.

\section{Author affiliations}

${ }^{1}$ National Centre for Sport and Exercise Medicine, School of Sport, Exercise and Health Sciences, Loughborough University, Loughborough, UK

${ }^{2}$ Department of Social Sciences, School of Social, Political and Geographical Sciences, Loughborough University

${ }^{3}$ Leicester-Loughborough Diet, Lifestyle and Physical Activity Biomedical Research Unit

${ }^{4}$ Centre for Exercise and Rehabilitation Science, Glenfield Hospital, University Hospitals of Leicester NHS Trust

Acknowledgements The authors would like to acknowledge the Patient and Public Involvement Group, University Hospitals of Leicester for providing feedback into the design and implementation of the trial. They thank Sally Schreder and the COPD Specialist Nurses for their advice and support throughout the design and implementation of the trial. The research took place at the University Hospitals of Leicester NHS Trust. Support was provided by the Collaboration for Leadership in Applied Health Research and Care East Midlands (CLAHRC-EM) and the NIHR Leicester Respiratory Biomedical Research Unit (BRU). The research was part funded by Loughborough University funding for our Centre for Doctoral Training in Chronic Diseases.

Contributors All authors of the paper have contributed to the design of the work, acquisition, analysis and interpretation of the data. MO, LS, DE, AW, PS and SS were involved in the development of the intervention and design of the trial. MO, LS, AW, PS and SS have been involved in drafting the work or revising it critically for important intellectual content. All authors have read and approved the final manuscript for publication (V3). 
Funding This work was funded by research facilitation funds from Loughborough University.

Disclaimer The views expressed are those of the authors and not necessarily those of the NHS, the NIHR, the Department of Health or Loughborough University.

\section{Competing interests None declared.}

Ethics approval Ethical approval has been received by the East Midlands Leicester Central Research Ethics Committee of the NHS Health Research Authority (Ethics reference number EM/15/0433).

Provenance and peer review Not commissioned; externally peer reviewed.

Data sharing statement Additional unpublished data are still being collected and analysed and are only available to members of the study team.

Open Access This is an Open Access article distributed in accordance with the Creative Commons Attribution Non Commercial (CC BY-NC 4.0) license which permits others to distribute, remix, adapt, build upon this work noncommercially, and license their derivative works on different terms, provided the original work is properly cited and the use is non-commercial. See: http:// creativecommons.org/licenses/by-nc/4.0/

\section{REFERENCES}

1. Watz $\mathrm{H}$, Pitta $\mathrm{F}$, Rochester $\mathrm{CL}$, et al. An official European Respiratory Society statement on physical activity in COPD. Eur Respir J 2014;44:1521-37.

2. Cindy Ng LW, Mackney J, et al. Does exercise training change physical activity in people with COPD? A systematic review and meta-analysis. Chron Respir Dis 2012;9:17-26.

3. Cavalheri V, Straker L, Gucciardi DF, et al. Changing physical activity and sedentary behaviour in people with COPD. Respirology 2016;21:419-26.

4. Hill K, Gardiner PA, Cavalheri V, et al. Physical activity and sedentary behaviour: applying lessons to chronic obstructive pulmonary disease. Intern Med 2015;45:474-82.

5. Sedentary Behaviour Research Network. Letter to the editor: standardized use of the terms "sedentary" and "sedentary behaviours". Appl Physiol Nutr Metab 2012;37:540-2.

6. Pitta F, Troosters T, Spruit MA, et al. Characteristics of physical activities in daily life in chronic obstructive pulmonary disease. Am J Respir Crit Care Med 2005;171:972-7.

7. Celli BR, Barnes PJ. Exacerbations of chronic obstructive pulmonary disease. Eur Respir J 2007;29:1224-38.

8. Suter P, Hennessey B, Florez D, et al. Review series: examples of chronic care model: the home-based chronic care model: redesigning home health for high quality care delivery. Chron Respir Dis 2011;8:43-52.

9. Morgan MD. Preventing hospital admissions for COPD: role of physical activity. Thorax 2003;58:95-6.

10. Puhan MA, Gimeno-Santos E, Scharplatz M, et al. Pulmonary rehabilitation following exacerbations of chronic obstructive pulmonary disease. Cochrane Database Syst Rev 2011;(10):CD005305.

11. Jones SE, Green SA, Clark AL, et al. Pulmonary rehabilitation following hospitalisation for acute exacerbation of COPD: referrals, uptake and adherence. Thorax 2014;69:181-2.

12. Greening NJ, Williams JE, Hussain SF, et al. An early rehabilitation intervention to enhance recovery during hospital admission for an exacerbation of chronic respiratory disease: randomised controlled trial. BMJ 2014;349:94315.

13. Pitta F, Troosters T, Probst VS, et al. Physical activity and hospitalization for exacerbation of COPD. Chest $J 2006 ; 129$ : $536-44$

14. Donaire-Gonzalez D, Gimeno-Santos E, Balcells E, et al. Benefits of physical activity on COPD hospitalisation depend on intensity. Eur Respir J 2015;46:1281-9.

15. Waschki B, Kirsten A, Holz O, et al. Physical activity is the strongest predictor of all-cause mortality in patients with COPD. Chest 2011;140:331-42.

16. Change in physical activity level following COPD exacerbation. Best abstracts in physical activity in chronic lung diseases, 2015. European Respiratory Society conference 2015; Abstract 3552. http://www.ers-education.org/events/international-congress/ amsterdam-2015 aspx?idParent=144785
17. Kawagoshi A, Kiyokawa N, Sugawara K, et al. Effects of low-intensity exercise and home-based pulmonary rehabilitation with pedometer feedback on physical activity in elderly patients with chronic obstructive pulmonary disease. Respir Med 2015;109: 364-71.

18. Mendoza L, Horta P, Espinoza J, et al. Pedometers to enhance physical activity in COPD: a randomised controlled trial. Eur Respir $J$ 2015;45:347-54.

19. Wicks P, Vaughan T, Heywood J. Subjects no more: what happens when trial participants realize they hold the power? BMJ 2014;348: g368.

20. Michie S, Abraham C, Whittington C, et al. Effective techniques in healthy eating and physical activity interventions: a meta-regression. Health Psychol 2009;28:690.

21. Carver CS, Scheier MF. Control theory: a useful conceptual framework for personality-social, clinical, and health psychology. Psychol Bull 1982;92:111.

22. Gardner B, Smith L, Lorencatto F, et al. How to reduce sitting time? A review of behaviour change strategies used in sedentary behaviour reduction interventions among adults. Health Psychol Rev 2015:10:1-24.

23. Campbell MK, Piaggio G, Elbourne DR, et al., CONSORT Group. Consort 2010 statement: extension to cluster randomised trials. BM 2012;345:e5661.

24. Gardner B, Thune-Boyle I, lliffe S, et al. 'On Your Feet to Earn Your Seat', a habit-based intervention to reduce sedentary behaviour in older adults: study protocol for a randomized controlled trial. Trials 2014;15:368.

25. Lally $\mathrm{P}$, Van Jaarsveld $\mathrm{CH}$, Potts HW, et al. How are habits formed: modelling habit formation in the real world. Eur J Soc Psychol 2010;40:998-1009.

26. Gardner B, Lally P, Wardle J. Making health habitual: the psychology of 'habit-formation' and general practice. Br J Gen Pract 2012;62:664-6.

27. Bellg AJ, Borrelli B, Resnick $B$, et al. Enhancing treatment fidelity in health behavior change studies: best practices and recommendations from the NIH Behavior Change Consortium. Health Psychol 2004;23:443.

28. Santacroce SJ, Maccarelli LM, Grey M. Intervention fidelity. Nurs Res 2004;53:63-6.

29. Freedson PS, Melanson E, Sirard J. Calibration of the computer science and applications, Inc. accelerometer. Med Sci Sports Exerc 1998:30:777-81.

30. Rosenberger ME, Buman MP, Haskell WL, et al. Twenty-four hours of sleep, sedentary behavior, and physical activity with nine wearable devices. Med Sci Sports Exerc 2016;48:457-65.

31. Kooiman TJ, Dontje ML, Sprenger SR, et al. Reliability and validity of ten consumer activity trackers. BMC Sports Sci Med Rehabil 2015;7:1

32. Jones PW, Harding G, Berry P, et al. Development and first validation of the COPD Assessment Test. Eur Respir $J$ 2009;34:648-54.

33. Herdman M, Gudex C, Lloyd A, et al. Development and preliminary testing of the new five-level version of EQ-5D (EQ-5D-5L). Qual Life Res 2011;20:1727-36.

34. Webster K, Cella D, Yost K. The Functional Assessment of Chronic IIIness Therapy (FACIT) Measurement System: properties, applications, and interpretation. Health Qual Life Outcomes 2003;1:79.

35. Zigmond AS, Snaith RP. The hospital anxiety and depression scale. Acta Psychiatr Scand 1983;67:361-70.

36. Yardley L, Beyer N, Hauer K, et al. Development and initial validation of the Falls Efficacy Scale-International (FES-I). Age Ageing 2005;34:614-19.

37. Marshall AL, Miller YD, Burton NW, et al. Measuring total and domain-specific sitting: a study of reliability and validity. Med Sci Sports Exerc 2010;42:1094-102.

38. Craig C, Marshall A, Sjöström M, et al. International Physical Activity Questionnaire (IPAQ): 12-country reliability and validity. Med Sci Sports Exerc 2003;35:81-95.

39. Mahler DA, Wells CK. Evaluation of clinical methods for rating dyspnea. Chest J 1988;93:580-6.

40. Puthoff ML. Research corner outcome measures in cardiopulmonary physical therapy: short physical performance battery. Cardiopulm Phys Ther J 2004;19:17-22.

41. Hudon C, Fortin M, Haggerty J, et al. Patient-centered care in chronic disease management: a thematic analysis of the literature in family medicine. Patient Educ Couns 2012;88:170-6.

42. Flick U. An introduction to qualitative research. Sage, 2009.

43. Glaser BG. The constant comparative method of qualitative analysis. Soc Probl 1965; 12:436-45. 


\section{Correction}

Orme M, Weedon A, Esliger D, et al. Study protocol for Chronic Obstructive Pulmonary Disease-Sitting and ExacerbAtions Trial (COPD-SEAT): a randomised controlled feasibility trial of a home-based self-monitoring sedentary behaviour intervention. BMJ Open 2016;6:e013014.

The middle initials for each author were not provided in the original submission. The author list with middle initials is:

Mark W Orme, Amie E Weedon, Dale W Esliger, Paula M Saukko, Mike D Morgan, Michael C Steiner, John W Downey, Sally J Singh, Lauren B Sherar

Open Access This is an Open Access article distributed in accordance with the Creative Commons Attribution Non Commercial (CC BY-NC 4.0) license, which permits others to distribute, remix, adapt, build upon this work noncommercially, and license their derivative works on different terms, provided the original work is properly cited and the use is non-commercial. See: http://creativecommons.org/licenses/by-nc/4.0/

BMJ Open 2016;6:e013014corr1. doi:10.1136/bmjopen-2016-013014corr1 